\title{
Manifestações digestórias em portadores de transtornos do espectro autístico necessidade de ampliar as perguntas e respostas
}

\author{
Autism spectrum disorders and possible connection with the digestive tract \\ Márcia Andrade Pinho' ${ }^{1}$, Luciana Rodrigues Silva ${ }^{2}$ \\ ${ }^{2}$ Doutoranda em Processos Interativos dos Órgãos e Sistemas, Instituto de Ciências da Saúde - Universidade \\ Federal da Bahia; ${ }^{2}$ Professor Titular do Departamento de Pediatria, Faculdade de Medicina da Bahia - \\ Universidade Federal da Bahia
}

\begin{abstract}
RESUMO
Objetivos: Descrever e analisar alterações gastrointestinais relacionadas com o sistema imunológico em crianças com transtornos do espectro autista que, muitas vezes, não são identificadas pelo pediatra na atenção primária. Metodologia: Consulta de artigos mais recentes nas bases Pubmed e Lilacs que abordem transtornos gastrointestinais, transtorno do espectro autístico, atenção primária, no período de $\mathbf{2 0 0 0}$ até 2011. Resultados: A idéia de uma fisiopatologia comum entre a doença do trato gastrointestinal e transtornos do espectro autista permanece controversa. Mais estudos precisam ser conduzidos e com maior rigor para definir a relação entre transtornos do espectro autista, trato gastrointestinal e sistema imune. Transtornos gastrointestinais em crianças com transtornos do espectro autista são tratáveis. Conclusão: É consenso na literatura a presença de comorbidades gastrointestinais e imunológicas em crianças com transtornos do espectro autista. Urge, portanto a construção de estratégias terapêuticas na atenção primária para identificá-las de forma a minimizar o impacto na qualidade de vida do paciente e da família.
\end{abstract}

Palavras-chave: Transtorno autístico. Gastroenteropatias. Doenças do sistema imune. Atenção primária à saúde.

\section{ABSTRACT}

Objectives: To describe and analyze gastrointestinal changes related to the immune system in children with autism spectrum disorders that often are not identified by the pediatrician in primary care. Methodology: Consultation of the latest articles in PubMed and Lilacs, to address gastrointestinal disorders, autistic spectrum disorder, primary care, between 2000 up to 2011. Results: The idea of a common pathophysiology between autism spectrum disorders and gastrointestinal disease remains controversial. More studies need to be conducted to better define the relationship between autism spectrum disorders, gastrointestinal tract and immune system. Gastrointestinal disorders in children with autism spectrum disorders are treatable. Conclusion: There is a consensus in the literature of the presence of gastrointestinal and immunologic comorbidities in children with autism spectrum disorders. It is therefore important to build therapeutic strategies in primary care to identify them in order to minimize the impact on quality of life of patients and their families.

Keywords: Autistic disorder. Gastrointestinal diseases. Immune system diseases. Primary health care.

\section{INTRODUÇÃO}

O transtorno do espectro autista (TEA) representa uma complexa desordem no neurodesenvolvimento, que traduz, na interação entre a suscetibilidade múltipla e variável de genes, efeitos epigenéticos e fatores ambientais. (HERBERT, 2010) e que pode se apresentar com variações na forma clínica e na gravidade.

Existe uma tendência atual a conceber o TEA como um conjunto de distúrbios da socialização com início precoce e curso crônico, cujas categorias nosológicas incluem condições associadas ao retardo mental (síndrome de Rett e transtorno desintegrativo da infância) e não associadas (autismo, transtorno global do desenvolvimento sem outra especificação), além de uma condição que é tipicamente associada à inteligência normal ou acima da média a Síndrome de Asperger. Os TEA caracterizam-se por um impacto variável em áreas

Recebido: 30 de setembro de 2011; revisado: 20 de dezembro de 2011 . Correspondência / Correspondence:Márcia Andrade Pinho. Rua Conselheiro Pedro Luiz, no 190, Rio Vermelho. 41950-610. Salvador, Bahia, Brasil. Tel.: (71) 3334-1532. E-mail: marcapinho@gmail.com múltiplas e nucleares do desenvolvimento, desde o estabelecimento da subjetividade, das relações pessoais, da linguagem, até o aprendizado e as capacidades adaptativas (BRYSON, ROGGERS; FOMBONNE, 2003; WING; POTTER, 2002). É importante salientar que a heterogeneidade etiológica, fenotípica e genotípica entre os indivíduos com TEA tem se associado às variações na expressão comportamental e aos vários níveis desta gravidade.

$\mathrm{Na}$ atualidade, estudos realizados na Ásia, Europa e Estados Unidos apontam para uma prevalência dos TEA de $0,6 \%$ a $1 \%$ na população, segundo dados do Centers for Disease Control and Prevention (2011). No Reino Unido, foram estimados $£ 2,7$ bilhões/ano em custos de suporte a crianças com TEA em 2009 (KNAPP, ROMEO; BEECHAM, 2009), o que demonstra o impacto econômico significativo produzido por esse transtorno.

Existe um consenso na literatura com relação à existência de interdependência funcional e interligação contínua entre o cérebro, o intestino e o sistema 
imunológico, visto que a homeostase funcional em todos os três sistemas é assegurada por substâncias múltiplas, tais como os hormônios, os neuropeptídeos, os neurotransmissores e as citocinas. Ademais, todos os três sistemas são imaturos ao nascer e requerem estímulos e interações ambientais apropriadas para que o desenvolvimento ocorra normalmente. $O$ cérebro requer estímulos sensoriais do meio ambiente, o sistema imunológico requer estímulos antigênicos e o intestino, por sua vez, necessita de colonização microbiana e de substrato alimentar, além da integridade morfológica e funcional. Estes estímulos interagem com outros fatores intrínsecos, genéticos e morfológicos do indivíduo para sua maturação adequada. A interdependência entre esses sistemas pode, eventualmente, traduzir uma agressão ambiental a um destes sistemas, que pode afetar indiretamente os outros dois, dependendo da gravidade e da duração da lesão.

Essa construção epistemiológica reforça a concepção dos TEA como um sistema multi-heterogêneo de condições que podem começar durante o desenvolvimento e ao longo deste, influenciado por diversos fatores externos (ambiente) e internos (comorbidades clínicas e psiquiátricas), os quais definiriam uma forma peculiar de expressão desta síndrome em cada indivíduo (fenótipo). A compreensão médica sobre a amplitude do TEA, portanto, mudou desde que o autismo foi definido por Kanner, em 1943, e nesse processo de reconceituação, foram identificadas algumas alterações clínicas que podem agravar o aspecto cognitivo e comportamental dessa população.

O objetivo deste trabalho é expor as alterações descritas da literatura que ocorrem no sistema gastrointestinal e suas interrelações com o sistema imunológico em crianças com TEA; que podem, muitas vezes, não ser identificadas como tal, na atenção primária, seja pela dificuldade em expressar da própria criança, seja pela dificuldade de identificação por parte do médico não especializado.

\section{COMORBIDADE GASTROINTESTINAL}

Na última década, o escopo na pesquisa do TEA ampliou-se para a exploração dos aspectos genéticos, ambientais, gastrointestinais, imunológicos e neurológicos que são apontados, atualmente, como fatores de risco para o desenvolvimento do transtorno. Investigações clínicas têm sugerido que as desordens no TEA, pelo menos em um dos subgrupos, estão presentes também em outros sistemas, dentre os quais o trato gastrointestinal (TGI). (HORVATH; PERMAN, 2002). Essa associação do TEA com algumas comorbidades clínicas tem sido demonstrada em alguns estudos, e talvez esteja associadas a fatores etiológicos na detrerminação do agravamento desta condição.

A conexão cérebro-intestinal tem sido reconhecida e o seu envolvimento com o sistema gastrointestinal respaldado em muitas das funções normais do intestino, bem como em alguns transtornos neuropsiquiatricos (HORVATH; PERMAN, 2002). Os quadros gastrointestinais mais descritos em pacientes portadores de TEA são: constipação, diarreia, dor abdominal, vômitos frequentes, disbiose, doença inflamatória intestinal, insuficiência pancreática exócrina, doença celíaca, intolerância alimentar, aumento de gases, padrão anormal das fezes, regurgitação de alimentos, seletividade por certos alimentos, refluxo gastroesofágico (RGE) e encoporese. A desregulação da resposta imune também tem sido descrita na literatura com potencial de resposta para patologias gastrointestinais nesses pacientes. (WAKEFIELD et al., 1998).

Apesar de já haver relatos anteriores sobre patologias do TGI em pacientes com TEA, apenas em 1971 foi publicado um estudo com 15 pacientes autistas selecionados aleatoriamente, dentre os quais foram descritas seis crianças com fezes volumosas, diarreia intermitente e uma com doença celíaca. O segundo estudo, feito em 1972, para avaliar doença celíaca em 15 crianças descreveu baixas concentrações séricas de alfa-1 antitripsina nas fazes em $53 \%$ da amostra. (WALKER-SMITH \& ANDREWS, 1972). Pouca atenção, porém, foi dispensada às conclusões desses estudos até alguns anos atrás, quando as avaliações gastrointestinais de rotina em crianças com autismo revelaram uma prevalência aumentada de sintomas gastrointestinais, alterações histológicas no trato digestório e disfunções gastrointestinais, quando comparadas com pacientes controles. (HORVATH; PERMAN, 2002).

A prevalência exata de sintomas gastroi-ntestinais em crianças com TEA é desconhecida. Atualmente, ainda é controversa a relação de TEA com sintomas gastrointestinais, mas, apesar das limitações, existem na literatura dois estudos que foram importantes na posterior concepção de pesquisas que relacionam o sistema gastrointestinal com o TEA. (WANG; TANCREDI ; THOMAS, 2011).

Em um estudo com 12 crianças diagnosticadas com autismo (todos com padrão regressivo), Wakefield et al. (1998) observaram uma variedade de doenças gastrointestinais, incluindo dor abdominal, diarreia e distensão abdominal, que foram extensivamente examinadas. Os sintomas do TGI desenvolvidos coincidiam com o comportamento inicial do autismo, de acordo com os pais. A endoscopia revelou que 10 das 12 crianças exibiam hiperplasia linfóide nodular no intestino (NHL, destas 12 crianças, oito apresentavam anormalidades na mucosa, na região composta por epitélio de absorção, tecido conjuntivo subjacente, camada muscular e mucosa. As anormalidades descritas na mucosa foram aumento de granulócitos, perda do padrão vascular e eritema irregular (colite inespecífica), resultados que foram confirmados por exames histológicos de biópsias da mucosa. A ressonância cerebral (RC) e o eletroencefalograma (EEG) não revelaram anormalidades neurológicas nessas crianças (WAKEFIELD et al., 2000) .

Em uma publicação mais recente, esses mesmos pesquisadores observaram um grupo de 60 crianças com vários distúrbios do desenvolvimento (WAKEFIELD et al., 2000), das quais 50 foram diagnosticadas com autismo (incluindo as 12 crianças do estudo original), $5 \mathrm{com}$ síndrome de Asperger (autismo sem retardo) e 2 com transtorno desintegrativo, uma outra subcategoria do TEA. Excetuando uma, dentre as 60 crianças, todas as outras tinham sintomas do TGI, incluindo dor abdominal, constipação, diarreia, distensão abdominal e refluxo 
gastroesofágico. Os resultados foram comparados com os de um grupo de 37 crianças com desenvolvimento normal (não-autistas) com sintomas do TGI semelhantes (o grupo controle). Observou-se: hiperplasia nodular linfóide do íleo em 93\% das crianças portadoras e em $14,3 \%$ das crianças do grupo controle; hiperplasia nodular linfóide do cólon, em $30 \%$ das crianças com TEA e 5,4\% das crianças controle; hiperplasia linfonodal do cólon, em $88,5 \%$ das biópsias de crianças com inflamação ativa do íleo (ileíte), em 8\%; e inflamação crônica do cólon (colite), em $88 \%$ das crianças afetadas.

Em outro estudo, Horvath et al. (1999) utilizaram endoscopia com biópsia para examinar o trato digestório de 36 crianças diagnosticadas com autismo que, concomitantemente, apresentavam dor abdominal, diarreia, dor crônica, distensão abdominal, dificuldades com o sono ou irritabilidade inexplicável. Achados anormais incluíram esofagite de refluxo em 25 das crianças, gastrite crônica em 15 , e duodenite crônica em 24 delas. Baixa atividade das enzimas digestivas intestinais para carboidratos foi observada em 21 crianças, enquanto 27 apresentaram aumento da secreção pancreática e da secreção biliar após a administração intravenosa do hormônio gastrointestinal secretina.

É importante notar que o último estudo referido descreve a função alterada no TGI superior de crianças autistas, enquanto que a hiperplasia linfóide nodular descrita por Wakefield et al. (1998; 2000) foi observada na porção mais baixa do intestino, no íleo e cólon. $O$ conjunto dos resultados desses diferentes estudos sugerem que importante fisiopatologia do TGI pode acompanhar os TEA, pelo menos, dentro de uma sub-população de pacientes. Assim, alguns pesquisadores sugerem que desordens do sistema gastrointestinal estariam envolvidas na etiologia de alguns subtipos de TEA, enquanto outros admitem, simplesmente uma associação secundária não casual . Em ambos os grupos, porém, os autores tendem a admitir a possibilidade de que tais patologias desempenhem um papel importante na sintomatologia apresentada pelos indivíduos com o espectro autista. (ASHWOOD; WILLS; VAN DE WATER, 2006).

Dois estudos retrospectivos em populações de crianças com autismo relataram sintomas do TGI em aproximadamente $20 \%$ das crianças, previamente diagnosticadas com autismo (FOMBONNE et al., 2001), fato que contrasta com estudos prospectivos na área da gastropediatria e da clínica geral, que têm descrito sintomas do TGI em $46-84 \%$ dos pacientes com TEA. (HORVATH et al., 1999).

Outras evidências de anormalidades funcionais do trato digestório em crianças com TEA descritas foram: baixa atividade de enzimas dissacaridases (HORVATH et al., 1999), crescimento bacteriano com maior diversidade e número de clostrídios (FINEGOLD et al., 2002), aumento da permeabilidade intestinal (D'EUFEMIA et al., 1996) e um efeito benéfico da exclusão do glúten ou da caseína sobre a cognição e o comportamento em alguns pacientes. (KNIVSBERG et al., 1995; KNIVSBERG et al., 2002).

No entanto, não existe um consenso sobre a prevalência dos sintomas gastrointestinais, bem como sobre a sua real interrelação com sintomas autistas, até o momento. Outro aspecto utilizado como argumento é a escassez de dados na literatura disponível e de estudos metodologicamente mais rigorosos realizados, com pacientes autistas que possam validar a presença de alterações do TGI associadas aos TEA. (ERICKSON et al., 2005).

\section{TGI EM PACIENTES COM TEA E ALTERAÇÕES DO SISTEMA IMUNE}

A mucosa intestinal representa a principal interface entre o sistema imunológico e o ambiente externo e seu desafio diário é manter a homeostase gastrointestinal. Na relação entre os TEA e os TGI, as alterações imunológicas podem contribuir e determinar com algumas desordens, cujas observações macroscópicas revelaram hiperplasia linfóide nodular (NHL) e achados histológicos de enterocolite. Uma das avaliações da realação quantitativas do TGI/imunidade é realizada através de dosagens dos níveis de citocinas pró-inflamatórias, as interleucinas (IL)6, IL-8, e IL-1, presentes em biópsias intestinais de crianças com TEA. (ASHWOOD; WILLS; VAN DE WATER, 2006).

De modo geral, as doenças inflamatórias intestinais são causadas por uma ativação intestinal inadequada do sistema imunológico que ocorre mediante uma superprodução de citocinas pró-inflamatórias com determinação de gravidade e extensão variável de sesão. Uma série de estudos tem mostrado que o grau de inflamação intestinal está correlacionado com upregulation de citocinas intestinais, especificamente IL-6, IL-8 e IL-1. De forma simplificada, o processo inflamatório se manifesta através de uma cascata de eventos da IL-6 secretada por monócitos ativados, que desempenha um papel central na estimulação e proliferação das células $T$ e na diferenciação terminal de células B (WAKEFIELD et al., 1998; WAKEFIELD et al., 2000). Uma variedade de tipos celulares, incluindo monócitos, fibroblastos, células epiteliais e produção de IL-8, que iniciam e dirigem a quimiotaxia de neutrófilos e IL-1, produzida por monócitos ativados, estão envolvidos na estimulação das células T e células $B$ e na produção de citocinas, prostaglandinas e óxido nítrico. (DEFELICE et al., 2003).

Algumas alterações na resposta imune foram relatadas em crianças autistas e incluem alterações nas células do tipo T helper 1 (TH1)/TH2, na diminuição do número de linfócitos, na diminuição da resposta mitógena de células $T$, no desequilíbrio dos níveis séricos de imunoglobulinas e nos perfis da citocinas que são responsáveis pela intensidade e duração da resposta imune. Tem sido sugerida, também, a associação dos TEA com doenças autoimunes, relacionadas aos antígenos leucocitários humanos (HLA)-DRB1 e complementar Alelo C4. (ASHWOOD; WILLS; VAN DE WATER, 2006).

O fator de crescimento transformador beta (TGF-b) tem sido associado aos TEA em vários estudos, haja vista que o TGF-b está envolvido em diversos aspectos do desenvolvimento, desde a migração celular, a apoptose e a regulação do sistema imunológico. (GOINES; VAN DE WATER, 2010).

Segundo Ashwood, Wills e Van De Water (2006), a atividade anormal do sistema imunológico, durante períodos cruciais do neurodesenvolvimento pode participar das alterações neurológicas características dos TEA, bem como da intensidade da resposta imunológica. 
Estudos clínicos têm descrito aspectos imunopatológicos nas desordens do TGI em crianças com TEA, como NHL crônica do íleo e enterocolite (PALMEN et al., 2004). Em ambas as alterações clínicas, as mucosas são descritas com um grau variável de inflamação e infiltrado de eosinófilos que sugerem possíveis processos imunológicos inflamatórios e/ou alérgicos. (WAKEFIELD et al., 1998).

A citometria de fluxo e as análises imunohistoquímicas dos linfócitos da mucosa nesse subgrupo de TEA demonstraram qualitativamente anormalidades consistentes em diferentes sítios anatômicos, incluindo estômago, duodeno, íleo e cólon, que indicam um padrão relativamente homogêneo de linfócitos nessas mucosas. Apesar de NHL não ser um achado incomum em crianças com alergia ou imunodeficiência, parece haver aumento da frequência e de gravidade em pacientes com autismo. (WAKEFIELD et al., 2000; PALMEN et al., 2004). Outros achados foram observados nesses estudos, como depósito de IgG e Cq1 na membrana basolateral dos enterócitos, esses achados, segundo os autores não foram encontrados na mucosa inflamada de crianças com desenvolvimento normal e em crianças com paralisia cerebral. (WAKEFIELD et al., 2000).

Embora exista um grande número de especu-lações, ainda não está claro qual o mecanismo que ocorre nas alterações da mucosa desses pacientes e de que forma essas podem influenciar no desenvolvimento ou no comportamento dos pacientes com TEA. Não obstante, é plausível que ocorra uma ativação imunológica primária decorrente de imunopatologia gastrointestinal, o que, por sua vez, poderia determinar um aumento da ativação imunológica sistêmica resultando em uma resposta inflamatória disseminada.

Tem sido descrita na doença celíaca imunopatologia da mucosa que ocorre como resultado da intolerância ao glúten o que, provavelmente, pode levar a algum quadro neuropatológico secundário, incluindo inflamação cerebral, demência, ataxia cerebelar, epilepsia e calcificações cerebrais heterotópicas. (ROBERTSON et al., 2008).

Uma conexão de doença celíaca foi proposta para explicar a presença de alguns sintomas em pacientes com TEA, nesse sentido, as alterações na permeabilidade gastrointestinal proporcionariam uma absorção de peptídeos pela degradação incompleta de proteínas como o glúten. Esses fragmentos de peptídeos, atravessando a barreira hemato-encefálica, promove-riam efeitos sobre o sistema nervoso central nos pacientes com TEA ou até poderiam determinar outros fênomenos de autoimunidade. Porém, até o presente, os resultados são conflitantes, não havendo consenso sobre a conexão doença celíaca e sintomas autísticos. Contudo, essa resposta imune, no epitélio da mucosa gastrointestinal, pode ser indicativo de um processo autoimune dirigido contra o antígeno nas células epiteliais, seguindo um processo inflamatório que pode alterar a função da barreira intestinal desses pacientes. (LIU; LI; NEU, 2005).

Finalmente, é importante ressaltar que o aumento de estudos sobre alterações imunológicas em indivíduos com TEA, tem demonstrado a crescente conscientização e suspeitas quanto ao papel desempenhado pelas desordens imunológicas na determinação de epifênomenos de, pelo menos, um dos subgrupos de pacientes com TEA.

\section{DISCUSSÃO}

Durante os últimos 25 anos, muito conhecimento foi produzido sobre os mecanismos neurobiológicos relacionados com o cérebro, mente e comportamentos do espectro autista. No entanto, foi agregado pouco conhecimento sobre o envolvimento de outros sistemas e de outros órgãos que podem ser importantes em crianças com TEA.

A identificação de alterações do TGI dessas crianças deve ser ampliada, pois poderá identificar fatores de risco, diminuindo, assim, o impacto negativo dos sintomas no processo das alterações do desenvolvimento comportamental. Outro aspecto significativo é a pesquisa de possíveis fatores genéticos importantes e/ou marcadores biológicos que, se identi-ficados, poderão refinar a capacidade na categorização clínica e dos subtipos dentro do amplo espectro do autismo. Há, portanto, muitas perguntas, ainda, a serem respondidas nas várias linhas de investigação neste campo.

No que tange os profissionais de saúde, sucede ainda uma grande dificuldade na identificação de sintomas clínicos sugestivos de TEA que podem, na maioria das vezes, estar relacionados com cooperação diminuída ou ausente dos pacientes, ou ainda, em função da sua limitação verbal (alguns são hipo ou não verbais) e de déficits no processamento sensorial, fato que os impedem de relatar com precisão a presença de dor ou a localização do desconforto ou de outros sintomas. Ademais, pacientes com TEA podem apresentar sintomas cuja semiologia não se enquadre nos quadros clínicos descritos na clínica pediátrica de crianças sem o TEA, o que pode dificultar também o diagnóstico adequado.

Muitos comportamentos de crianças com TEA, tais como, agressão, auto-agressividade, inquietação e insônia, têm sido atribuídos apenas a quadros comportamentais ou a alterações no quadro psiquiátrico dos pacientes, dirigindo as ações no campo da saúde para itinerários terapêuticos centrados em intervenções psicofarmacológicas. Nesse aspecto, parece que a dificuldade da interpretação desses comportamentos como indicadores de dor e/ou desconforto gastrointestinal, que acometeria qualquer criança, dá lugar a um conjunto de significados que são interpretados como sofrimento psíquico ao invés de outra condição clínica subjacente orgânica localizada no trato digestório (HORVATH; PERMAN, 2002; ALVES; RABELO, 1998).

A operacionalização desse complexo diagnóstico/ tratamento de transtornos gastrointes-tinais em crianças com TEA exige ainda outro registro importante, o registro ético, da equidade do direito à saúde, como qualquer outra criança assistida na atenção primária do sistema de saúde, o que significa que, independentemente das questões etiológicas ligando TEA a transtornos gastrointestinais, se existirem sintomas, esses precisam ser avaliados, diagnosticados e tratados como quaisquer outras crianças.

Vale salientar ainda que alguns estudos assinalaram a importância da avaliação do uso de medicações psiquiátricas que podem também causar 
alterações gastrointestinais, pois alguns psicofármacos utilizados no controle dos sintomas comportamentais podem ser potentes deflagradores de sintomas o TGI (GONZALEZ, 2005).

Em estudo realizado, com crianças com TEA, Horvath e Perman (2002) observaram que 52\% das crianças tinham sintomas gastrintestinais e apresentavam perturbações do sono, e aquelas que tinham esofagite de refluxo exibiram irritabilidade inexplicável com mais frequência (43\%) do que as que não tinham esses distúrbios (13\%).

Outro aspecto relevante que merece menção é que o diagnóstico de alterações clínicas em crianças com TEA encerra um significado na qualidade de vida da criança e, principalmente para a família, que já lida com a cronicidade dos sintomas comportamentais da síndrome, fato que pode contribuir com desordens na interação entre os membros da família.

Até o momento, há ainda poucos e bem concebidos estudos populacionais adequadamente desenhados e realizados, investigando qualquer dos vários aspectos que fazem parte da discussão envolvendo desordens pediátricas associadas ao TEA, o que nos permite considerar o papel da atenção primária como de fundamental importância na avaliação e diagnóstico desses sintomas clínicos (GOLNIK; IRELAND; BOROWSKY, 2009). Não obstante, alguns trabalhos vêm apontando a necessidade da atenção primária prestar atendimentos a essa clientela com uma melhor qualidade, com mais resolutividade e menos fragmentação na assistência (PINTO-MARTIN et al., 2008; THOMAS; MORRISSEY; MCLAURIN, 2007; GOLNIK; IRELAND; BOROWSKY, 2009), buscando principalmente a assistência multidisciplinar, que é a ideal para estes pacientes.

No campo da saúde mental para crianças e adolescentes, recomendações recentes da Organização Mundial da Saúde (OMS) chamam a atenção para a importância da articulação de uma rede assistencial em prol de uma cobertura da atenção integral. No entanto, parece que a atenção integral da criança com transtornos mentais ainda representa um desafio na organização do atendimento cotidiano. Segundo Leckman e Leventhal (2008), o estigma associado ao transtorno mental e o número reduzido de profissionais treinados, e adequadamente instruídos para lidar com esta condição traduzem a necessidade urgente de desenvolver intervenções eficazes visando a implementação de melhorias na atenção integral a essa população. Essa necessidade é mais bem percebida quando se evidencia que as etiologias da maioria dos transtornos mentais da infância, e não apenas os TEA, são complexos e multifatoriais, o que também não é diferente no que tange à grande maioria das condições clínicas que afetam essa população. Estudo recente constatou que crianças autistas e com retardo têm maior dificuldade no acesso à prestação de serviços básicos de saúde do que os portadores de deficiências físicas. (LECKMAN; LEVENTHAL, 2008).

No Brasil, ainda constitui um desafio para o Programa de Saúde da Família a construção e a consolidação de uma "rede pública ampliada" para a atenção integral e intersetorial a crianças e adolescentes com transtornos mentais, que seja capaz de conjugar acesso com qualidade de assistência. (FERRIOLLI; MARTURANO; PUNTEL, 2007).

Em estudo realizado com médicos da atenção primária da American Medical Association Physicians Masterfile, nos EUA, foram encontrados os seguintes dados: os médicos da atenção primária não se sentem competentes para realizar o diagnóstico de pacientes com TEA e muito menos para identificar alterações clínicas nessa população. O reconhecimento por parte dos médicos da atenção primária é mais precária em relação a crianças com TEA do que as crianças com desordens neurológicas, doenças crônicas e condições médicas complexas de outras etiologias. Assim, existe uma necessidade de melhorar a educação médica e a capacitação de médicos e outros profissionais de saúde que atuam na atenção primária a fim de minimizar as deficiências nesse setor, foi outro achado importante no estudo. (GOLNIK; IRELAND; BOROWSKY, 2009).

Em consultas realizadas às bases de dados PUBMED e LILACS, não foram encontrados no Brasil estudos que tratassem da atenção integral para crianças com TEA ou de comorbidades clínicas ou gastrointestinais. Dado o impacto do tema, também na literatura foi encontrado um número reduzido de publicações em toda América Latina (GONZÁLEZ, 2005; GONZÁLEZ et al., 2006).

$O$ exame da realidade do Brasil, no que concerne a assistência dos TEA, existem os Centros de Atenção Psicossocial da Infância (CAPSi) que representam hoje a principal estratégia na assistência à saúde mental da infância. Porém, até 2009, existiam apenas 101 CAPSi para suprir toda a demanda de assistência no grande território brasileiro. É importante salientar que, com a criação dos CAPSi (2002), os pacientes que se encontravam fora do sistema de saúde como, por exemplo, os com TEA, passaram a ser formalmente assistidos pelo SUS, no entanto ainda de modo inadequado (BRASIL, 2010).

Hoje na Bahia existem quatro unidades CAPSi para dar conta da assistência em saúde mental de crianças e adolescentes incluindo aquelas com TEA. Teoricamente, caberia a esse dispositivo, articular a assistência intersetorial dessa população e a multidisciplinaridade, algo que até o momento não tem ocorrido. Entretanto, é interessante destacar que a Lei Estadual nh? 10.553, de 23 de março de 2007 (BAHIA, 2007) impõe ao Governo do Estado garantir assistência integral às pessoas com autismo e transtornos globais do desenvolvimento, na rede do SUS, e, ainda, fomentar a capacitação de seus profissionais para esse atendimento, além de outras estratégias contempladas pela lei, como a construção de rede especializada, na rede territorializada no SUS-Ba.

Parece ser provável a presença de alterações gastrointestinais e imunológicas nos pacientes portadores de TEA e a constatação do impacto desses quadros na qualidade de vida da criança e da família. Há, portanto ampla necessiade de estudos a serem realizados nesta área. Atualmente, no Brasil, especificamente, na Bahia, ao lado da ausência de estudos e da falta de estratégias capazes de articular essa assistência, ainda existem questionamentos sem resposta: existem crianças com TEA no Estado da Bahia que tem alterações gastrointestinais? Quais as alterações mais prevalentes nestes pacientes? Existe algum protocolo para avaliar as alterações 
gastrointestinais e imunopatológicas existentes nestas crianças? Quais as estratégias diagnósticas e terapêuticas utilizadas pelos médicos e outros profissionais de saúde na atenção básica para essas patologias do TGI, em crianças com TEA?

\section{CONCLUSÃO}

É consenso na literatura a existência de comorbidades associadas em pacientes portadores dos TEA com a presença de sintomas gastrointestinais. Muitas dessas alterações têm sido ignoradas, em parte, porque encerram vários desafios que vão desde o exame físico, que pode ser difícil de executar nestes pacietnes, investimentos em estudos para melhor definir a relação de sintomas específicos dos TEA às patologias do TGI, a necessidade de melhor preparar os profissionais de saúde que desenvolvem atividades com estes pacientes em atividades multidisciplinares, até a vigência real de uma política de saúde que possa efetivamente garantir uma atenção integral a essa população de pacientes com TEA.

\section{REFERÊNCIAS}

ALVES, P. C.; RABELO, M. C. (Orgs.). Antropologia da Saúde: traçando identidade e explorando fronteiras. Rio de Janeiro: Relume Dumará; Fiocruz, 1998.

ASHWOOD, P.; WILLS, S.; VAN DE WATER, J. The immune response in autism: a new frontier for autism research. J. Leukoc. Biol., WinstonSalem, v. 80, n. 1, p. 1-15, Jul. 2006.

BAHIA. Lei n. 10.553, de 23 de março de 2007. Determina a obrigatoriedade do Governo do Estado da Bahia proporcionar tratamento especializado, educação e assistência específicas a todos os autistas do Estado, independentemente de idade. Diário Oficial do Estado da Bahia, Salvador, BA, 23 mar. 2007. Disponível em: <http://www.egba.ba.gov.br/doonline/doonline.asp>. Acesso em: 20 maio de 2011.

BRASIL. Ministério da Saúde. Número de Centros de Atenção Psicossocial (CAPS) por tipo e UF e Indicador CAPS/100.000 habitantes: Brasil - dezembro de 2010. Brasília. Disponível em: <http://portal.saude.gov.br/portal/arquivos/pdf/

2011_1_21caps_uf_dez.pdf>. Acesso em: 18 maio de 2011.

BRYSON, S.; ROGERS, S. J.; FOMBONNE, E. Autism spectrum disorders: early detection, intervention, education, and psychopharmacological management. Can. J. Psychiatry, Ottawa, v. 48, n. 8, p. 506-516, Sep. 2003.

CENTERS FOR DISEASE CONTROL AND PREVENTION. Autism Spectrum Disorders (ASDs), Atlanta, 14 nov. 2011. Disponível em: <http:// www.cdc.gov/ncbddd/autism/index.html >. Acesso em: 20 maio de 2011.

D'EUFEMIA, P. et al. Abnormal intestinal permeability in children with autism. Acta Paediatr., Stockholm, v. 85, n. 9, p. 1076-1079, Sep. 1996.

DEFELICE, M. L. et al. Intestinal cytokines in children with pervasive developmental disorders. Am. J. Gastroenterol., New York, v. 98, n. 8, p. 1777-1782, Aug. 2003.

ERICKSON, C. A. et al. Gastrointestinal factors in autistic disorder: a critical review. J. Autism Dev. Disord., New York, v. 35, n. 6, p. 713-727, Dec. 2005.

FERRIOLLI, S. H.; MARTURANO, E. M.; PUNTEL, L. P. Contexto familiar e problemas de saúde mental infantil no Programa Saúde da Família. Rev. Saúde Pública, São Paulo, v. 41, n. 2, p. 251-259, abr. 2007.

FINEGOLD, S. M. et al. Gastrointestinal microflora studies in lateonset autism. Clin. Infect. Dis., Chicago, v. 35, suppl. 1, p. S6-S16, Sep. 2002.

FOMBONNE, E. et al. Prevalence of pervasive developmental disorders in the British nationwide survey of child mental health.
J. Am. Acad. Child Adolesc. Psychiatry, Baltimore, v. 40, n. 7, p. 820827, Jul. 2001.

GOINES, P.; VAN DE WATER, J. The immune system's role in the biology of autism. Curr. Opin. Neurol., Philadelphia, v. 23, n. 2, p. 111-117, Apr. 2010.

GOLNIK, A.; IRELAND, M.; BOROWSKY, I. W. Medical homes for children with autism: a physician survey. Pediatrics, Evanston, v. 123, n. 3, p. 966-971, Mar. 2009.

GONZÁLEZ, L. G. et al; Características endoscópicas, histológicas e inmunológicas de la mucosa digestiva en niños autistas con síntomas gastrointestinales. Arch. Venez. Pueric. Pediatr., Caracas, v. 69, n. 1, p. 19-25, ene. 2006.

GONZÁLEZ, L. G. Manifestaciones gastrointestinales en trastornos del espectro autista. Colomb. Méd., Cali, v. 36, n. 2, supl. 1, p. 3638, abr. 2005.

HERBERT, M. R. Contributions of the environment and environmentally vulnerable physiology to autism spectrum disorders. Curr. Opin. Neurol., Philadelphia, v. 23, n. 2, p. 103-110, Apr. 2010.

HORVATH, K. et al. Gastrointestinal abnormalities in children with autistic disorder. J. Pediatr., St. Louis, v. 135, n. 5, p. 559-563, Nov. 1999.

HORVATH, K; PERMAN, J. A. Autism and gastrointestinal symptoms. Curr. Gastroenterol. Rep., Philadelphia, v. 4, n. 3, p. 251-258, Jun. 2002.

KNAPP, M.; ROMEO, R.; BEECHAM, J. Economic cost of autism in the UK. Autism, London, v. 13, n. 3, p. 317-336, May 2009.

KNIVSBERG A. M. et al. A randomised, controlled study of dietary intervention in autistic syndromes. Nutr. Neurosci., Amsterdam, v. 5, n. 4, p. 251-261, Sep. 2002.

KNIVSBERG A. M. et al. Autistic syndromes and diet: a follow-up study. Scand. J. Educ. Res., Oslo, v. 39, n. 3, p. 222-236, 1995.

LECKMAN, J. F.; LEVENTHAL, B. L. Editorial: a global perspective on child and adolescent mental health. J. Child Psychol. Psychiatry, Elmsford, v. 49, n. 3, p. 221-225, Mar. 2008.

LIU, Z.; LI, N.; NEU, J. Tight junctions, leaky intestines, and pediatric diseases. Acta Paediatr., Stockholm, v. 94, n. 4, p. 386393, Apr. 2005.

PALMEN, S. J. et al. Neuropathological findings in autism. Brain, London, v. 127, pt. 12, p. 2572-2583, Dec. 2004.

PINTO-MARTIN, J. A. et al. Screening strategies for Autism Spectrum Disorders in pediatric primary care. J. Dev. Behav. Pediatr., Baltimore, v. 29, n. 5, p. 345-350, Oct. 2008.

ROBERTSON, M. A. et al. Intestinal permeability and glucagon-like peptide-2 in children with autism: a controlled pilot study. J. Autism Dev. Disord., New York, v. 38, n. 6, p. 1066-1071, Jul. 2008.

THOMAS, K. C., MORRISSEY, J. P., MCLAURIN, C. Use of autismrelated services by families and children. J. Autism Dev. Disord., New York, v. 37, n. 5, p. 818-829, May 2007.

WAKEFIELD A. J. et al. Ileal-lymphoid-nodular hyperplasia, nonspecific colitis, and pervasive developmental disorder in children. Lancet, London, v. 351, n. 9103, p. 637-641, Feb. 1998.

WAKEFIELD, A. J. et al. Enterocolitis in children with developmental disorders. Am. J. Gastroenterol., New York, v. 95, n. 9, p. 2285-2295, Sep. 2000.

WALKER-SMITH J.; ANDREWS, J. Alpha-1-antitrypsin, autism, and coeliac disease. Lancet, London, v. 2, n. 7782, p. 883-884, Oct. 1972.

WANG, L. W.; TANCREDI, D. J.; THOMAS, D. W. The prevalence of gastrointestinal problems in children across the United States with autism spectrum disorders from families with multiple affected members. J. Dev. Behav. Pediatr., Baltimore, v. 32, n. 5, p. 351-360, Jun. 2011.

WING, L.; POTTER, D. The epidemiology of autistic spectrum disorders: is the prevalence rising? Ment. Retard. Dev. Disabil. Res. Rev., New York, v. 8, n. 3, p. 151-161, 2002. 